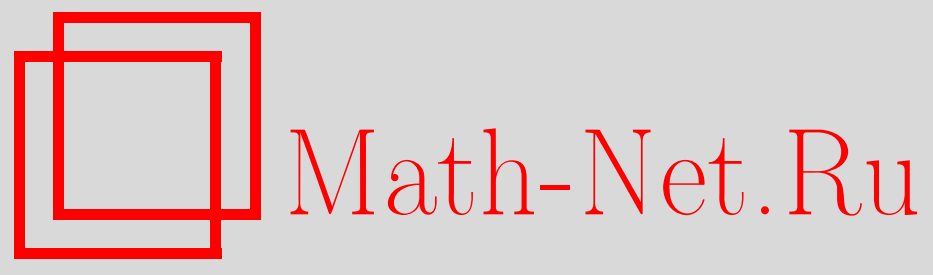

В. М. Круглов, Усиление арксинус-неравенства Прохорова, Теория вероятн. и ее примен., 2005, том 50, выпуск 4, 767-774

DOI: https://doi.org/10.4213/tvp130

Использование Общероссийского математического портала Math-Net.Ru подразумевает, что вы прочитали и согласны с пользовательским соглашением

http: //www . mathnet.ru/rus/agreement

Параметры загрузки:

IP : 54.205 .225 .156

26 апреля 2023 г., 12:43:42

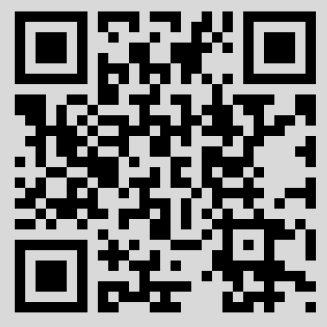


Следовательно, $\mathbf{P}\{\xi>\eta\}$, где $\xi=\mathbf{E}\left(Y_{\tau} \mid \mathscr{F}_{\tau \wedge \sigma}\right)$ и $\eta=\mathbf{E}\left(Z_{\sigma} \mid \mathscr{F}_{\tau \wedge \sigma}\right)$. Предположим сначала, что $\mathbf{P}(\{\xi>\eta\} \cap\{\tau \leqslant \sigma\})>0$. Тогда на множестве $\{\tau \leqslant \sigma\}$ мы имеем

$$
\xi=Y_{\tau}=Y_{\tau \wedge \sigma}, \quad \eta=\mathbf{E}\left(Z_{\sigma} \mid \mathscr{F}_{\tau \wedge \sigma}\right)=\mathbf{E}\left(Z_{\tau \vee \sigma} \mid \mathscr{F}_{\tau \wedge \sigma}\right),
$$

что противоречит предположению леммы. Аналогичное противоречие возникает в случае $\mathbf{P}(\{\xi>\eta\} \cap\{\tau \geqslant \sigma\})>0$. Тем самым, мы показали, что $\widetilde{Y} \leqslant \widetilde{Z}$. Сушествование искомого процесса $M$ теперь следует из [1, лемма 3] (приведенное авторами доказательство справедливо только для непрерывных справа с пределами слева процессов, однако с небольшими изменениями его можно распространить на общий случай).

\section{СПИСОК ЛІИТЕРАТУРЫ}

1. Jouini E., Kallal H. Martingales and arbitrage in securities markets with transaction costs. - J. Econom. Theory, 1995, v. 66, № 1, p. 178-197.

2. El Karoui $N$. Les aspects probabilistes du controle stochastique. - Lecture Notes in Math., 1981, v. 876, p. 73-238.

3. Revuz D., Yor M. Continuous Martingales and Brownian Motion. Berlin: SpringerVerlag, 1999, $602 \mathrm{p}$.

Поступила в редакцию

31.III. 2005

(c) 2005 r.

КРУГЛОВ В. М.*

\section{УСИЛЕНИЕ АРКСИНУС-НЕРАВЕНСТВА ПРОХОРОВА ${ }^{1)}$}

Доказано усиление известного неравенства Прохорова для сумм независимых равномерно ограниченных случайных величин. Аналогичное неравенство доказано для хвостов безгранично делимых функций распределения, спектральные функции которых сосредоточены на некотором сегменте.

Ключевые слова и фразы: случайная величина, мартингал-разность, фильтрация, безгранично делимая функция распределения, спектральная функция.

Неравенство Прохорова, приведенное ниже под первым номером, доказано в статье [1]. Оно обладает высокой точностью и определенной красотой. Возможно, эти качества послужили поводом для авторов монографии [2] сказать (с. 372), что «в случае сумм равномерно ограниченных независимых случайных величин неравенство Прохорова уточняет неравенство Бернштейна и является наилучшим из возможных неравенств». Тем не менее, неравенство Прохорова можно усилить. Например, правая часть неравенства (2) примерно совпадает с квадратом правой части неравенства (1). Более высокая точность достигается усложнением вида неравенства.

Оценка (2) совпадает с оценками (13) хвостов безгранично делимых функций распределения, спектральные меры которых сосредоточены на конечном сегменте. Такая оценка становится возможной благодаря тому, что каждая такая функция распределения является слабым пределом функций распределения сумм равномерно ограниченных независимых случайных величин.

* Московский государственный университет им. М.В. Ломоносова, факультет вычислительной математики и кибернетики, Ленинские горы, 119992 Москва, Россия; e-mail: vkrug@dataforce.net

1) Работа выполнена при поддержке Российского фонда фундаментальных исследований, гранты № 05-01-00535, 05-01-00583. 
В упомянутой монографии [2] и в статьях [3] и [4] доказаны варианты неравенства Прохорова для мартингалов. Наиболее общий вариант неравенства содержится в монографии [2, с. 372]. Его доказательство основано на использовании метода декаплинга, состояшего в том, что моменты некоторых функций от зависимых случайных величин можно оценить посредством моментов этих функций от специально построенных случайных величин, условно независимых относительно некоторой сигмаалгебры. Доказанное ниже неравенство (5) сильнее всех упомянутых неравенств. При этом для его доказательства потребовались более скромные средства, а именно, начальные сведения из теории мартингалов. Любопытно, что в идейном плане все необходимое для доказательства более точного неравенства содержится в упомянутой статье Ю. В. Прохорова [1].

1. Независимые случайные величины. Пусть независимые случайные величины $X_{1}, \ldots, X_{n}$ определены на вероятностном пространстве $(\Omega, \mathscr{F}, \mathbf{P})$. Предположим, что $\left|X_{k}\right| \leqslant c, \mathbf{E} X_{k}=0$ для всех $k=1, \ldots, n$ и для некоторого числа $c>0$, $\sigma^{2}=\mathbf{E} X_{1}^{2}+\cdots+\mathbf{E} X_{n}^{2}>0$. Обозначим $S_{k}=X_{1}+\cdots+X_{k}, k=1, \ldots, n$.

Неравенство Прохорова. Если случайные величины $X_{1}, \ldots, X_{n}$ удовлетворяют перечисленным условиям, то для любого $x \geqslant 0$

$$
\mathbf{P}\left\{S_{n}>x\right\} \leqslant \exp \left\{-\frac{x}{2 c} \operatorname{Arcsh}\left(\frac{x c}{2 \sigma^{2}}\right)\right\},
$$

где $\operatorname{Arcsh} x=\ln \left(x+\sqrt{x^{2}+1}\right)-$ гиперболический арксинус - функиия, обратная $\kappa$ гиперболическому синусу $\operatorname{sh} x=\left(e^{x}-e^{-x}\right) / 2, x \in \mathbf{R}=(-\infty, \infty)$.

По следствию 1 , доказанному далее, справедливо более сильное утверждение

$$
\mathbf{P}\left\{\max _{1 \leqslant k \leqslant n} S_{k}>x\right\} \leqslant \exp \{-Q(x, c, \sigma)\} \leqslant \exp \left\{-\frac{x}{2 c} \operatorname{Arcsh}\left(\frac{x c}{2 \sigma^{2}}\right)\right\},
$$

где

$$
Q(x, c, \sigma)=\frac{x}{c} \operatorname{Arcsh}\left(\frac{x c}{2 \sigma^{2}}\right)-\frac{2 \sigma^{2}}{c^{2}}\left(\operatorname{ch}\left(\operatorname{Arcsh}\left(\frac{x c}{2 \sigma^{2}}\right)\right)-1\right),
$$

$\operatorname{ch} x=\left(e^{x}+e^{-x}\right) / 2, x \in \mathbf{R},-$ гиперболический косинус.

Неотрицательная функция $Q(x, c, \sigma), x \geqslant 0$, неограниченно возрастает и

$$
\lim _{x \rightarrow \infty} \frac{Q(x, c, \sigma)}{x c^{-1} \operatorname{Arcsh}\left(x c / 2 \sigma^{2}\right)}=1 \text {. }
$$

Второе неравенство в (2), на самом деле, является строгим неравенством для $x>0$. Достаточно доказать неравенство

$$
\frac{x}{2 c} \operatorname{Arcsh}\left(\frac{x c}{2 \sigma^{2}}\right)<Q(x, c, \sigma)
$$

для всех $x>0$. Обозначим $y=x c / 2 \sigma^{2}$. Предполагаемое неравенство принимает следуюший вид: $0<y \operatorname{Arcsh} y-2 \operatorname{ch}(\operatorname{Arcsh} y)+2$ для $y>0$. Первые две производные функции $\psi(y)=y \operatorname{Arcsh} y-2 \operatorname{ch}(\operatorname{Arcsh} y)+2$ равны $\psi^{\prime}(y)=\operatorname{Arcsh} y-y(\operatorname{Arcsh} y)^{\prime}$ и $\psi^{\prime \prime}(y)=-y(\operatorname{Arcsh} y)^{\prime \prime}=y^{2}\left(1+y^{2}\right)^{-3 / 2}$. Так как $\psi^{\prime \prime}(y)>0$ и $\psi^{\prime}(0)=0$, то $\psi^{\prime}(y)>0$ для всех $y>0$. Поэтому функьия $\psi$ строго возрастает. Так как $\psi(0)=0$, то $\psi(y)>0$ для всех $y>0$.

Неравенство (2) уместно сравнить с неравенством Беннета-Хёфдинга [5]-[7]

$$
\mathbf{P}\left\{S_{n}>x\right\} \leqslant \exp \left\{\frac{x}{c}-\left(\frac{x}{c}+\frac{\sigma^{2}}{c^{2}}\right) \ln \left(\frac{x c}{\sigma^{2}}+1\right)\right\} .
$$

Оно имеет определенное сходство с неравенством Прохорова. Для сравнительно небольших $x$ неравенства (2) и (3) менее точны по сравнению с неравенством Колмогорова (см. $[8$, с. 36$])$,

$$
\mathbf{P}\left\{\max _{1 \leqslant k \leqslant n} S_{k}>x\right\} \leqslant \begin{cases}\exp \left\{-\frac{x^{2}}{2 \sigma^{2}}\left(1-\frac{x c}{2 \sigma^{2}}\right)\right\}, & \text { если } 0 \leqslant x c \leqslant \sigma^{2}, \\ \exp \left\{-\frac{x}{4 c}\right\}, & \text { если } \quad x c \geqslant \sigma^{2} .\end{cases}
$$


В оригинальном неравенстве Колмогорова вместо $\max _{1 \leqslant k \leqslant n} S_{k}$ стоит $S_{n}$. Справедливость (4) доказана в следствии 2 ниже.

С целью сравнить правые части неравенств (2) и (4) мы докажем, что

$$
Q\left(x, c, \sigma^{2}\right) \leqslant \frac{x^{2}}{2 \sigma^{2}}\left(1-\frac{x c}{2 \sigma^{2}}\right), \quad \text { если } 0 \leqslant x c \leqslant \sigma^{2} .
$$

Воспользуемся старым обозначением $y=x c / 2 \sigma^{2}$. Неравенство эквивалентно неравенству $y^{2}(1-y)-y \operatorname{Arcsh} y+\operatorname{ch}(\operatorname{Arcsh} y)-1 \geqslant 0$ для $0 \leqslant y \leqslant \frac{1}{2}$. Первые три производные функции $\phi(y)=y^{2}(1-y)-y \operatorname{Arcsh} y+\operatorname{ch}(\operatorname{Arcsh} y)-1$ равны

$$
\begin{gathered}
\phi^{\prime}(y)=2 y-3 y^{2}-\ln \left(y+\sqrt{y^{2}+1}\right), \quad \phi^{\prime \prime}(y)=2-6 y-\left(y^{2}+1\right)^{-1 / 2}, \\
\phi^{\prime \prime \prime}(y)=-6+y\left(y^{2}+1\right)^{-3 / 2} .
\end{gathered}
$$

Заметим, что $\phi(0)=0, \phi\left(\frac{1}{2}\right)>0, \phi^{\prime}(0)=0, \phi^{\prime}\left(\frac{1}{2}\right)<0, \phi^{\prime \prime}(0)>0, \phi^{\prime \prime}\left(\frac{1}{2}\right)<0$, $\phi^{\prime \prime \prime}(y)<0$. Отсюда следует, что функция $\phi$ принимает неотрицательные значения на сегменте $\left[0, \frac{1}{2}\right]$.

Для сравнения (2) и (4) нам также понадобятся неравенства

$$
Q(x)\left\{\begin{array}{lll}
\leqslant \frac{x}{4 c}, & \text { если } & \sigma^{2} \leqslant x c \leqslant 2 y_{0} \sigma^{2}, \\
\geqslant \frac{x}{4 c}, & \text { если } \quad x c \geqslant 2 y_{0} \sigma^{2},
\end{array}\right.
$$

где $y_{0}-$ корень уравнения $y \ln \left(y+\sqrt{y^{2}+1}\right)-\sqrt{y^{2}+1}+1-y / 4=0$. Заметим, что $\frac{1}{2}<y_{0}<1$. Мы снова воспользуемся обозначением $y=x c / 2 \sigma^{2}$. Первое неравенство эквивалентно неравенству $y / 4-y \operatorname{Arcsh} y+\operatorname{ch}(\operatorname{Arcsh} y)-1 \geqslant 0$ для $\frac{1}{2} \leqslant y \leqslant y_{0}$. Первые две производные функции $p(y)=y / 4-y \operatorname{Arcsh} y+\operatorname{ch}(\operatorname{Arcsh} y)-1$ равны $p^{\prime}(y)=1 / 4-$ $\ln \left(y+\sqrt{y^{2}+1}\right)$ и $p^{\prime \prime}(y)=-\left(y^{2}+1\right)^{-1 / 2}$. Так как $p^{\prime \prime}(y)<0$ и $p^{\prime}\left(\frac{1}{2}\right)<0$, то $p^{\prime}(y) \leqslant 0$ и, следовательно, функция $p(y)$ убывает. Так как $p\left(\frac{1}{2}\right)>0$ и $p\left(y_{0}\right)=0$, то $p(y) \geqslant 0$ для $y \in\left[\frac{1}{2}, y_{0}\right]$. Попутно было доказано второе неравенство. Из сказанного следует, что

$$
\mathbf{P}\left\{\max _{1 \leqslant k \leqslant n} S_{k}>x\right\} \leqslant\left\{\begin{array}{lll}
\exp \left\{-\frac{x^{2}}{2 \sigma^{2}}\left(1-\frac{x c}{2 \sigma^{2}}\right)\right\}, & \text { если } 0 \leqslant x c \leqslant \sigma^{2}, \\
\exp \left\{-\frac{x}{4 c}\right\}, & \text { если } \sigma^{2}<x c \leqslant 2 y_{0} \sigma^{2}, \\
\exp \{-Q(x, c, \sigma)\}, & \text { если } & x c>2 y_{0} \sigma^{2} .
\end{array}\right.
$$

2. Мартингал-разности. Пусть случайные величины $X_{1}, \ldots, X_{n}$ определены на вероятностном пространстве $(\Omega, \mathscr{F}, \mathbf{P}),\left|X_{k}\right| \leqslant c$ для всех $k=1, \ldots, n$ и для некоторого числа $c>0$. В отличие от предыдушего случая мы будем предполагать, что случайные величины $X_{1}, \ldots, X_{n}$ образуют мартингал-разность относительно некоторого возрастающего набора сигма-алгебр $\mathscr{F}_{1}, \ldots, \mathscr{F}_{n} \subseteq \mathscr{F}$, или, как принято говорить, относительно фильтрации $\mathscr{F}_{1}, \ldots, \mathscr{F}_{n}$. Последнее означает, что для каждого $k=1, \ldots, n$ случайная величина $X_{k}$ измерима относительно $\mathscr{F}_{k}$ и условное математическое ожидание $\mathbf{E}\left(X_{k+1} \mid \mathscr{F}_{k}\right)$ равно нулю почти всюду (п.в.) по отношению к вероятности $\mathbf{P}$. Отсюда, в частности, следует, что $\mathbf{E} X_{k+1}=\mathbf{E}\left(\mathbf{E} X_{k+1} \mid \mathscr{F}_{k}\right)=0$. Предположим дополнительно, что $\mathbf{E} X_{1}=0$. Введем следующие обозначения: $S_{k}=X_{1}+\cdots+X_{k}$, $k=1, \ldots, n, \mathscr{F}_{0}$ - сигма-алгебра, состоящая из пустого множества $\varnothing$ и пространства элементарных событий $\Omega, B_{k}^{2}=\sum_{m=1}^{k} \mathbf{E}\left(X_{m}^{2} \mid \mathscr{F}_{m-1}\right), k=1, \ldots, n$.

Теорема 1. Если случайные величины $X_{1}, \ldots, X_{n}$ удовлетворяют перечисленньм условиям, то для любых чисел $x \geqslant 0$ и $\sigma^{2}>0$

$$
\mathbf{P}\left\{\bigcup_{k=1}^{n}\left\{S_{k}>x, B_{k}^{2} \leqslant \sigma^{2}\right\}\right\} \leqslant \exp \{-Q(x, c, \sigma)\} .
$$

Следствие 1. Если выполнень условия теоремь 1 u $B_{n}^{2} \leqslant \sigma^{2}$ n.в., то $\mathbf{P}\left\{\max _{1 \leqslant k \leqslant n} S_{k}>x\right\} \leqslant \exp \{-Q(x, c, \sigma)\}$. 
В случае независимых случайных величин в качестве $\mathscr{F}_{k}$ следует взять сигмаалгебру $\sigma\left(X_{1}, \ldots, X_{k}\right)$, порожденную случайными величинами $X_{1}, \ldots, X_{k}$. Тогда $B_{n}^{2}=\mathbf{E} X_{1}^{2}+\cdots+\mathbf{E} X_{n}^{2}$. Следствие выполняется с $\sigma^{2}=B_{n}^{2}$.

Д ок аза т ел ь с т в о те о ре мы 1. Достаточно рассмотреть случай $x>0$. Положим

$$
\tau=\left\{\begin{array}{lll}
\min \left\{k: 1 \leqslant k \leqslant n, S_{k}>x\right\} & \text { на множестве } & \left\{\max _{1 \leqslant k \leqslant n} S_{k}>x\right\}, \\
n+1 & \text { на множестве } & \left\{\max _{1 \leqslant k \leqslant n} S_{k} \leqslant x\right\} .
\end{array}\right.
$$

Случайная величина $\tau$ является марковским моментом относительно фильтрации $\mathscr{F}_{1}, \ldots, \mathscr{F}_{n}$, другими словами, для любого $k=1, \ldots, n$ множество $\{\tau=k\}$ принадлежит сигма-алгебре $\mathscr{F}_{k}$. На множестве $A=\bigcup_{k=1}^{n}\left\{S_{k}>x, B_{k}^{2} \leqslant \sigma^{2}\right\}$ выполняются соотношения $\tau \leqslant n, S_{\tau \wedge n}=S_{\tau}>x, B_{\tau \wedge n}^{2}=B_{\tau}^{2} \leqslant \sigma^{2}$, где $\tau \wedge n=\min \{\tau, n\}$. Поэтому для любого $\lambda>0$

$$
\mathbf{P}(A) \leqslant \mathbf{P}\left\{S_{\tau \wedge n}>x, B_{\tau \wedge n} \leqslant \sigma^{2}\right\}=\mathbf{P}\left\{e^{\lambda S_{\tau \wedge n}}>e^{\lambda x}, B_{\tau \wedge n}^{2} \leqslant \sigma^{2}\right\} .
$$

Далее нам понадобится следуюшее тождество:

$$
e^{\lambda S_{\tau \wedge n}}=e^{\lambda S_{\tau \wedge n}}\left[\prod_{j=1}^{\tau \wedge n} \mathbf{E}\left(e^{\lambda X_{j}} \mid \mathscr{F}_{j-1}\right)\right]^{-1}\left[\prod_{j=1}^{\tau \wedge n} \mathbf{E}\left(e^{\lambda X_{j}} \mid \mathscr{F}_{j-1}\right)\right] .
$$

Оценим последний из трех сомножителей справа в (7). С этой целью мы воспользуемся известными неравенствами $\alpha \leqslant e^{\alpha-1}$ и $e^{\alpha}-1-\alpha \leqslant 2(\operatorname{ch} \alpha-1)$, справедливыми для любого $\alpha \in \mathbf{R}$, и условием $\mathbf{E}\left(X_{j} \mid \mathscr{F}_{j-1}\right)=0$ п.в. для всех $j=1, \ldots, n$. В результате мы получим

$$
\begin{aligned}
& \prod_{j=1}^{\tau \wedge n} \mathbf{E}\left(e^{\lambda X_{j}} \mid \mathscr{F}_{j-1}\right) \leqslant \prod_{j=1}^{\tau \wedge n} \exp \left\{\mathbf{E}\left(e^{\lambda X_{j}} \mid \mathscr{F}_{j-1}\right)-1\right\} \\
& \quad=\exp \left\{\sum_{j=1}^{\tau \wedge n} \mathbf{E}\left(e^{\lambda X_{j}}-1-\lambda X_{j} \mid \mathscr{F}_{j-1}\right)\right\} \leqslant \exp \left\{2 \sum_{j=1}^{\tau \wedge n} \mathbf{E}\left(\operatorname{ch}\left(\lambda X_{j}\right)-1 \mid \mathscr{F}_{j-1}\right)\right\} .
\end{aligned}
$$

Положим $f(\alpha)=(\operatorname{ch} \alpha-1) \alpha^{-2}$ для $\alpha \neq 0$ и $f(0)=\frac{1}{2}$. Функция $f$ непрерывна на вещественной прямой и четна. Она возрастает на положительной полупрямой, так как ее производная $f^{\prime}(\alpha)=(\alpha \operatorname{sh} \alpha-2(\operatorname{ch} \alpha-1)) \alpha^{-3}$ принимает неотрицательные значения для всех $\alpha>0$. Убедимся в этом. Первые две производные функции $\lambda(\alpha)=$ $\alpha \operatorname{sh} \alpha-2 \operatorname{ch} \alpha+2$ равны $\lambda^{\prime}(\alpha)=\alpha \operatorname{ch} \alpha-\operatorname{sh} \alpha$ и $\lambda^{\prime \prime}(\alpha)=\alpha \operatorname{sh} \alpha$. Так как $\lambda^{\prime \prime}(\alpha) \geqslant 0$ и $\lambda^{\prime}(0)=0$, то $\lambda^{\prime}(\alpha) \geqslant 0$. Поэтому функция $\lambda(\alpha)$ возрастает. Так как $\lambda(0)=0$, то $\lambda(\alpha) \geqslant 0$ и, следовательно, $f^{\prime}(\alpha) \geqslant 0$ для всех $\alpha>0$.

Так как $\left|X_{j}\right| \leqslant c$ для всех $j=1, \ldots, n$, то

$$
\operatorname{ch}\left(\lambda X_{j}\right)-1=\left(\operatorname{ch}\left(\lambda X_{j}\right)-1\right)\left(\lambda X_{j}\right)^{-2}\left(\lambda X_{j}\right)^{2} \leqslant(\operatorname{ch}(\lambda c)-1) c^{-2} X_{j}^{2}
$$

и, следовательно,

$$
\prod_{j=1}^{\tau \wedge n} \mathbf{E}\left(e^{\lambda X_{j}} \mid \mathscr{F}_{j-1}\right) \leqslant \exp \left\{2(\operatorname{ch}(\lambda c)-1) \sum_{j=1}^{\tau \wedge n} \mathbf{E}\left(X_{j}^{2} \mid \mathscr{F}_{j-1}\right)\right\}=\exp \left\{2(\operatorname{ch}(\lambda c)-1) B_{\tau \wedge n}^{2}\right\} .
$$

Привлекая (6) и (7), мы получим

$$
\begin{aligned}
\mathbf{P}(A) & \leqslant \mathbf{P}\left\{e^{\lambda S_{\tau \wedge n}}\left[\prod_{j=1}^{\tau \wedge n} \mathbf{E}\left(e^{\lambda X_{j}} \mid \mathscr{F}_{j-1}\right)\right]^{-1} e^{2(\operatorname{ch}(\lambda c)-1) c^{-2} B_{\tau \wedge n}}>e^{\lambda x}, B_{\tau \wedge n} \leqslant \sigma^{2}\right\} \\
& \leqslant \mathbf{P}\left\{e^{\lambda S_{\tau \wedge n}}\left[\prod_{j=1}^{\tau \wedge n} \mathbf{E}\left(e^{\lambda X_{j}} \mid \mathscr{F}_{j-1}\right)\right]^{-1} e^{2(\operatorname{ch}(\lambda c)-1) c^{-2} \sigma^{2}}>e^{\lambda x}\right\} \\
& \leqslant \mathbf{E}\left(e^{\lambda S_{\tau \wedge n}}\left[\prod_{j=1}^{\tau \wedge n} \mathbf{E}\left(e^{\lambda X_{j}} \mid \mathscr{F}_{j-1}\right)\right]^{-1}\right) e^{-\lambda x+2(\operatorname{ch}(\lambda c)-1) c^{-2} \sigma^{2}} .
\end{aligned}
$$


На последнем этапе мы воспользовались неравенством Маркова. Убедимся, что математическое ожидание в правой части (8) равно единице. С этой целью заметим, что случайные величины $T_{k}=e^{\lambda S_{k}}\left[\prod_{j=1}^{k} \mathbf{E}\left(e^{\lambda X_{j}} \mid \mathscr{F}_{j-1}\right)\right]^{-1}, k=1, \ldots, n$, образуют мартингал относительно фильтрации $\mathscr{F}_{1}, \ldots, \mathscr{F}_{n}$. Действительно, для каждого $k=1, \ldots, n$ случайная величина $T_{k}$ измерима относительно сигма-алгебры $\mathscr{F}_{k}$, имеет конечное математическое ожидание и

$$
\mathbf{E}\left(T_{k+1} \mid \mathscr{F}_{k}\right)=\mathbf{E}\left[T_{k} \mathbf{E}\left(e^{\lambda X_{k+1}}\left[\mathbf{E}\left(e^{\lambda X_{k+1}} \mid \mathscr{F}_{k}\right)\right]^{-1} \mid \mathscr{F}_{k}\right)\right]=T_{k} \quad \text { п.в. }
$$

Случайные величины $T_{\tau \wedge k}, k=1, \ldots, n$, также образуют мартингал относительно фильтрации $\mathscr{F}_{1}, \ldots, \mathscr{F}_{n}$. Поэтому $\mathbf{E} T_{\tau \wedge n}=\mathbf{E} T_{1}=1$. Отсюда и из (8) следует, что $\mathbf{P}(A) \leqslant \exp \left\{-\lambda x+2 c^{-2}(\operatorname{ch}(\lambda c)-1) \sigma^{2}\right\}$. Показатель в экспоненте принимает свое минимальное значение $-Q(x, c, \sigma)$ при $\lambda=(x / c) \operatorname{Arcsh}\left(x c / 2 \sigma^{2}\right)$. Теорема доказана.

Доказываемое ниже неравенство (9) сильнее неравенства (5) при малых значениях $x$. Оно представляет собой обобщение неравенства Колмогорова $[8$, с. 36].

Теорема 2. Если случайные величины $X_{1}, \ldots, X_{n}$ удовлетворяют перечисленньци условиям, то для любьх чисел $x \geqslant 0 u \sigma^{2}>0$

$$
\mathbf{P}\left\{\bigcup_{k=1}^{n}\left\{S_{k}>x, B_{k}^{2} \leqslant \sigma^{2}\right\}\right\} \leqslant \begin{cases}\exp \left\{-\frac{x^{2}}{2 \sigma^{2}}\left(1-\frac{x c}{2 \sigma^{2}}\right)\right\}, & \text { eсли } 0 \leqslant x c \leqslant \sigma^{2}, \\ \exp \left\{-\frac{x}{4 c}\right\}, & \text { eсли } \quad x c \geqslant \sigma^{2} .\end{cases}
$$

Д о к а з а т ел ь с т в о. Так как $\left|X_{j}\right| \leqslant c$, то

$$
\mathbf{E}\left(\left|X_{j}\right|^{s} \mid \mathscr{F}_{j-1}\right) \leqslant c^{s-2} \mathbf{E}\left(X_{j}^{2} \mid \mathscr{F}_{j-1}\right) \text { п.в. }
$$

для $j=1, \ldots, n$ и $s=2,3, \ldots$ Для $0 \leqslant \lambda c \leqslant 1$ мы имеем

$$
\begin{gathered}
\mathbf{E}\left(e^{\lambda X_{j}} \mid \mathscr{F}_{j-1}\right)=1+\sum_{s=2}^{\infty} \frac{\lambda^{s} \mathbf{E}\left(X_{j}^{s} \mid \mathscr{F}_{j-1}\right)}{s !} \leqslant 1+\frac{\lambda^{2} \mathbf{E}\left(X_{j}^{2} \mid \mathscr{F}_{j-1}\right)}{2} \sum_{s=2}^{\infty} \frac{(\lambda c)^{s-2}}{s !} \\
\leqslant 1+\frac{\lambda^{2} \mathbf{E}\left(X_{j}^{2} \mid \mathscr{F}_{j-1}\right)}{2}\left(1+\frac{\lambda c}{2}\right) \leqslant \exp \left\{\frac{\lambda^{2} \mathbf{E}\left(X_{j}^{2} \mid \mathscr{F}_{j-1}\right)}{2}\left(1+\frac{\lambda c}{2}\right)\right\} \text { п.в. }
\end{gathered}
$$

Отсюда следует, что

$$
\prod_{j=1}^{\tau \wedge n} \mathbf{E}\left(e^{\lambda X_{j}} \mid \mathscr{F}_{j-1}\right) \leqslant \exp \left\{\frac{\lambda^{2}}{2} B_{\tau \wedge n}^{2}\left(1+\frac{\lambda c}{2}\right)\right\} \text { п.в. }
$$

Привлекая (6) и (7), так же, как при доказательстве теоремы 1, мы получим

$$
\begin{aligned}
\mathbf{P}(A) & \leqslant \mathbf{P}\left\{e^{\lambda S_{\tau \wedge n}}\left[\prod_{j=1}^{\tau \wedge n} \mathbf{E}\left(e^{\lambda X_{j}} \mid \mathscr{F}_{j-1}\right)\right]^{-1} \exp \left\{\frac{1}{2} \lambda^{2} \sigma^{2}\left(1+\frac{\lambda c}{2}\right)\right\}>e^{\lambda x}\right\} \\
& \leqslant \mathbf{E}\left(e^{\lambda S_{\tau \wedge n}}\left[\prod_{j=1}^{\tau \wedge n} \mathbf{E}\left(e^{\lambda X_{j}} \mid \mathscr{F}_{j-1}\right)\right]^{-1}\right) \exp \left\{-\lambda x+\frac{1}{2} \lambda^{2} \sigma^{2}\left(1+\frac{\lambda c}{2}\right)\right\} \\
& =\exp \left\{-\lambda x+\frac{1}{2} \lambda^{2} \sigma^{2}\left(1+\frac{\lambda c}{2}\right)\right\} .
\end{aligned}
$$

Полагая $\lambda=x / \sigma^{2}$ в случае $x c \leqslant \sigma^{2}$ и $\lambda=1 / c$ в случае $x c \geqslant \sigma^{2}$, мы придем к (9). Теорема доказана.

Следствие 2. Если выполнены условия теоремы 2 и $B_{n}^{2} \leqslant \sigma^{2}$ n.в., то

$$
\mathbf{P}\left\{\max _{1 \leqslant k \leqslant n} S_{k}>x\right\} \leqslant \begin{cases}\exp \left\{-\frac{x^{2}}{2 \sigma^{2}}\left(1-\frac{x c}{2 \sigma^{2}}\right)\right\}, & \text { если } 0 \leqslant x c \leqslant \sigma^{2}, \\ \exp \left\{-\frac{x}{4 c}\right\}, & \text { если } \quad \text { xс } \geqslant \sigma^{2} .\end{cases}
$$

Комментарии об условии $B_{n}^{2} \leqslant \sigma^{2}$ в следствии 1 остаются в силе. 
3. Безгранично делимые распределения. Функция распределения $F$ называется безгранично делимой, если ее характеристическая функция $f$ при любом натуральном $n$ является $n$-й степенью некоторой характеристической функции $f_{n}$. Она может быть представлена (см. $[9$, с. 91]) в следуюшем виде:

$$
f(t)=\exp \left\{i t \gamma-\frac{\delta^{2}}{2} t^{2}+\int_{-\infty}^{0} K(x, t) d M(x)+\int_{0}^{\infty} K(x, t) d N(x)\right\}, \quad t \in \mathbf{R},
$$

где $\gamma \in \mathbf{R}, \delta \in[0, \infty), K(x, t)=e^{i t x}-1-i t x /\left(1+x^{2}\right)$, неубываюшие функции $M$ и $N$ определены на $(-\infty, 0)$ и $(0, \infty)$, непрерывны слева, $M(-\infty)=N(\infty)=0$,

$$
\int_{-\varepsilon}^{0} x^{2} d M(x)+\int_{0}^{\varepsilon} x^{2} d N(x)<\infty \quad \text { для любого } \varepsilon>0 .
$$

3 а м е ч а н и е. Если математическое ожидание $a$ и дисперсия $\sigma^{2}$ безгранично делимой функции распределения $F$ сушествуют, то

$$
\begin{aligned}
a & =\int_{\infty}^{\infty} x d F(x)=\gamma+\int_{-\infty}^{0} \frac{x^{3}}{1+x^{2}} d M(x)+\int_{0}^{\infty} \frac{x^{3}}{1+x^{2}} d N(x), \\
\sigma^{2} & =\int_{\infty}^{\infty}(x-a)^{2} d F(x)=\delta^{2}+\int_{-\infty}^{0} x^{2} d M(x)+\int_{0}^{\infty} x^{2} d N(x)
\end{aligned}
$$

В этом можно убедиться с помощью дифференцирования функции (10). Функции $M$ и $N$ часто называют спектральными функциями.

Теорема 3. Пусть $F$ - безгранично делимая функиия распределения с характеристической функиией (10), $M(x)=0$ для $x<-c u N(x)=0$ для $x>c$ для некоторого числа $c>0$. Тогда величины (11) и (12) существуют $и$

$$
\begin{array}{rlrl}
F(x) \leqslant \exp \{-Q(a-x, c, \sigma)\} & \partial\lrcorner \mathcal{A} & x \leqslant a, \\
1-F(x) \leqslant \exp \{-Q(x-a, c, \sigma)\} & \text { dлs } \quad x \geqslant a .
\end{array}
$$

Д о к аз а т е л с т в о. Сначала мы сведем задачу к случаю, когда функции $M$ и $N$ имеют ограниченные вариации. Для любого натурального числа $n$ определим функции $M_{n}$ и $N_{n}$, положив $M_{n}(x)=M(x)$ для $x \leqslant-1 / n$ и $M_{n}(x)=M(-1 / n)$ для $-1 / n<x<0 ; N_{n}(x)=N(1 / n)$ для $0<x \leqslant 1 / n$ и $N_{n}(x)=N(x)$ для $x>1 / n$. Формула (10) с $M_{n}$ и $N_{n}$ вместо $M$ и $N$ определяет безгранично делимую функцию распределения $F_{n}$. Последовательность $\left\{F_{n}\right\}_{n \geqslant 1}$ слабо сходится к $F$ и $\lim _{n \rightarrow \infty} a_{n}=a$ и $\lim _{n \rightarrow \infty} \sigma_{n}^{2}=\sigma^{2}$, где $a_{n}$ и $\sigma_{n}^{2}-$ математическое ожидание и дисперсия функции распределения $F_{n}$. Если неравенства (13) справедливы для $F_{n}$, то с помощью предельного перехода при $n \rightarrow \infty$ мы придем к неравенствам (13) для $F$.

Далее предполагается, что $M(0-)=\lim _{x \uparrow 0} M(x)<\infty$ и $N(0+)=\lim _{x \downarrow 0} N(x)>$ $-\infty$. Определим функцию $F_{n}$, положив

$$
F_{n}(x)=\left\{\begin{array}{lll}
\frac{M(x)}{n} & \text { для } \quad x<0, \\
\frac{M(0-)}{n} & \text { для } \quad x=0, \\
1+\frac{N(x)}{n} & \text { для } \quad x>0 .
\end{array}\right.
$$

Для всех $n$, начиная с некоторого $n_{0}$, функция $F_{n}$ монотонно возрастает и, следовательно, является функцией распределения. На некотором вероятностном пространстве $(\Omega, \mathscr{F}, \mathbf{P})$ можно построить независимые случайные величины $\xi_{n 1}, \ldots, \xi_{n n}$, $\eta_{n 1}, \ldots, \eta_{n n}$ такие, что $\xi_{n k}$ имеет своей функцией распределения $F_{n}$, а $\eta_{n k}$ имеет равномерное распределение на $[-1,1], k=1, \ldots, n$.

По центральной предельной теореме (см. $[9$, с. 194]), для любого $x \in \mathbf{R}$

$$
\lim _{n \rightarrow \infty} \mathbf{P}\left\{\delta\left(\eta_{n 1}+\cdots+\eta_{n n}\right)<x \sqrt{\frac{n}{3}}\right\}=\frac{1}{\sqrt{2 \pi \delta^{2}}} \int_{-\infty}^{x} e^{-u^{2} / 2 \delta^{2}} d u
$$


где функция справа при $\delta=0$ совпадает с функцией распределения, вырожденной в нуле. Пусть $G$ обозначает функцию распределения с характеристической функцией

$$
g(t)=\exp \left\{i t \gamma+\int_{-\infty}^{0} K(x, t) d M(x)+\int_{0}^{\infty} K(x, t) d N(x)\right\}, \quad t \in \mathbf{R} .
$$

Убедимся, что при некотором выборе чисел $b_{n} \in \mathbf{R}$

$$
\lim _{n \rightarrow \infty} \mathbf{P}\left\{\xi_{n 1}+\cdots+\xi_{n n}+b_{n}<x\right\}=G(x)
$$

для любого $x \in \mathbf{R}$, являющегося точкой непрерывности функции $G$. Для этого достаточно убедиться, что выполняются четыре условия известной теоремы [9, c. 124]. В рассматриваемом случае они сводятся к следуюшим соотношениям:

$$
\begin{aligned}
& \lim _{n \rightarrow \infty} \max _{1 \leqslant k \leqslant n} \mathbf{P}\left\{\left|\xi_{n k}\right|>\varepsilon\right\}=\lim _{n \rightarrow \infty} \frac{1}{n}(M(-\varepsilon)-N(\varepsilon)=0 \quad \text { для любого } \varepsilon>0, \\
& \lim _{n \rightarrow \infty} \sum_{k=1}^{n} \mathbf{P}\left\{\xi_{n k}<x\right\}=M(x), \quad x<0, \\
& \lim _{n \rightarrow \infty} \sum_{k=1}^{n} \mathbf{P}\left\{\xi_{n k} \geqslant x\right\}=N(x), \quad x>0 \\
& \lim _{\varepsilon \downarrow 0} \limsup _{n \rightarrow \infty} \sum_{k=1}^{n}\left[\int_{|x|<\varepsilon} x^{2} d \mathbf{P}\left\{\xi_{n k}<x\right\}-\left(\int_{|x|<\varepsilon} x d \mathbf{P}\left\{\xi_{n k}<x\right\}\right)^{2}\right] \\
& \leqslant \lim _{\varepsilon \downarrow 0}\left[\int_{-\varepsilon}^{0} x^{2} d M(x)+\int_{0}^{\varepsilon} x^{2} d N(x)\right]=0
\end{aligned}
$$

для любого $x>0$ такого, что $-x$ и $x$ являются точками непрерывности функций $M$ и $N$. Тем самым (15) доказано.

Обозначим $X_{n k}=\xi_{n k}-\mathbf{E} \xi_{n k}, k=1, \ldots, n, X_{n k}=\eta_{n k} \sqrt{3 \delta^{2} / n}, k=n+1, \ldots, 2 n$, $a_{n}=b_{n}+\mathbf{E} \xi_{n 1}+\cdots+\mathbf{E} \xi_{n n}, S_{n}=X_{n 1}+\cdots+X_{n, 2 n}$. Для любого $\varepsilon>0$ и для всех достаточно больших $n$ выполняются неравенства $3 \delta^{2} \leqslant c^{2} n$ и

$$
\max _{1 \leqslant k \leqslant n}\left|\mathbf{E} \xi_{n k}\right|=\frac{1}{n}\left|\int_{-\infty}^{0} x d M(x)+\int_{0}^{\infty} d N(x)\right|<\varepsilon .
$$

Можно считать, что эти неравенства выполняются для всех $n \geqslant n_{0}$. Для таких $n$ случайные величины $X_{n k}, k=1, \ldots, 2 n$, имеют нулевые математические ожидания и равномерно ограничены числом $c+\varepsilon$. По неравенству (2) для любого $x>a_{n}$

$$
\mathbf{P}\left\{a_{n}+S_{n}>x\right\} \leqslant \exp \left\{-Q\left(x-a_{n}, c+\varepsilon, \sigma_{n}\right)\right\},
$$

где

$\sigma_{n}^{2}=\mathrm{E} S_{n}^{2}=\delta^{2}+\int_{-\infty}^{0} x^{2} d M(x)+\int_{0}^{\infty} x^{2} d N(x)-\frac{1}{n}\left(\int_{-\infty}^{0} x d M(x)+\int_{0}^{\infty} x^{2} d N(x)\right)^{2}$.

Из (14)-(16) следует, что $\lim _{n \rightarrow \infty} a_{n}=a, \lim _{n \rightarrow \infty} \sigma_{n}^{2}=\sigma^{2}$ и

$$
1-F(x)=\lim _{n \rightarrow \infty} \mathbf{P}\left\{a_{n}+S_{n}>x\right\} \leqslant \exp \{-Q(x-a, c+\varepsilon, \sigma)\}
$$

для любого $x>a$, являюшегося точкой непрерывности функции $F$ и, следовательно, для любого $x>a$. Полагая $\varepsilon \downarrow 0$, придем ко второму неравенству в (13). Доказательство первого неравенства сводится к доказательству второго неравенства. Действительно, пусть $\xi$ - случайная величина с функцией распределения $F$. Тогда $F(x)=\mathbf{P}\{\xi<x\}=\mathbf{P}\{-\xi>-x\}$. Все предыдушие рассуждения применимы к безгранично делимой функции распределения случайной величины $-\xi$. Теорема доказана.

3 а м е ч а н и е. В [10] доказано, что условие

$$
\lim _{x \rightarrow \infty} \frac{\ln (F(-x)+1-F(x))}{x \ln x}=\frac{1}{c}
$$

необходимо и достаточно для того, чтобы спектральные функции $M$ и $N$ безгранично делимой функции $F$ были сосредоточены на сегменте $[-c, c]$. Отсюда следует, что оценки (13) являются асимптотически (при $|x| \rightarrow \infty$ ) точными. 


\section{СПИСОК ЛИТЕРАТУРЫ}

1. Прохоров Ю.В. Одна экстремальная задача теории вероятностей. - Теория вероятн. и ее примен., 1959 , т. 4, в. 2, с. 211-214.

2. de la Peña V. H., Giné E. Decoupling. New York: Springer-Verlag, 1999, 392 p.

3. Levental S. A. A uniform CLT for uniformly bounded families of martingale differences. - J. Theoret. Probab., 1989, v. 2, № 3, p. 271-287.

4. Hitczenko P. Best constants in martingale version of Rosenthal's inequality. - Ann. Probab., 1990, v. 18, № 4, p. 1656-1668.

5. Bennett $G$. Probability inequalities for the sum of independent random variables. J. Amer. Statist. Assoc., 1962, v. 57, p. 33-45.

6. Bennett $G$. On the probability of large deviations from the expectation for sums of bounded, independent random variables. - Biometrika, 1963, v. 50, p. 528-535.

7. Hoeffding $W$. Probability inequalities for sums of bounded random variables. J. Amer. Statist. Assoc., 1963, v. 58, № 301, p. 13-30.

8. Колмогоров A. H. Теория вероятностей и математическая статистика. М.: Наука, $1986,534 \mathrm{c}$

9. Гнеденко Б. В., Колмогоров А. Н. Предельные распределения для сумм независимых случайных величин. М.-Л.: Гостехиздат, 1949, 264 с.

10. Круглов В. М., Антонов С. Н. Об асимптотическом поведении безгранично делимых распределений в банаховом пространстве. - Теория вероятн. и ее примен., 1982 , т. 27 , в. 4 , с. $625-642$.

Поступила в редакцию 8.XI.2005

(C) $2005 \Gamma$.

КУЗНЕЦОВА А. Я.*

\section{О СХОДИМОСТИ К РАВНОМЕРНОМУ РАСПРЕДЕЛЕНИЮ}

Рассматриваются суммы независимых одинаково распределенных случайных величин. Приводится пример, в котором при неограниченном росте числа слагаемых плотности вероятностей $p_{n}(x)$ дробных частей этих сумм сходятся к единице в смысле

$$
\int_{-\infty}^{\infty}\left|\widetilde{p}_{n}(x)-1\right| d x \rightarrow 0,
$$

но не сходятся к единице в равномерной метрике

$$
\sup _{0 \leqslant x \leqslant 1}\left|\tilde{p}_{n}(x)-1\right| .
$$

Ключевые слова и фразы: дробные части, случайные величины, равномерные распределения, сходимость «по вариации».

Пусть $X_{1}, X_{2}, \ldots, X_{n}, \ldots-$ независимые одинаково распределенные случайные величины и $S_{n}=X_{1}+\cdots+X_{n}$. Предположим, что $\mathbf{E} X_{k}=0, \mathbf{D} X_{k}=\sigma^{2}$ и что существует плотность $p\left(x ; X_{k}\right)$. Рассмотрим распределение дробной части $\left\{S_{n}\right\}$. Мы приводим пример, в котором плотность $p\left(x ;\left\{S_{n}\right\}\right)$ не сходится в равномерном смысле к 1 на отрезке $[0,1]$, но распределение $\left\{S_{n}\right\}$ сходится к равномерному на $[0,1]$ распределению по вариации. Этот пример напоминает пример, указанный в 446 книги [1], но как в русском издании, так и в английском [2] имеется неточность, которую мы здесь исправляем.

* Московский государственный университет им. М. В. Ломоносова, факультет вычислительной математики и кибернетики, Ленинские горы, 119992 Москва, Россия. 